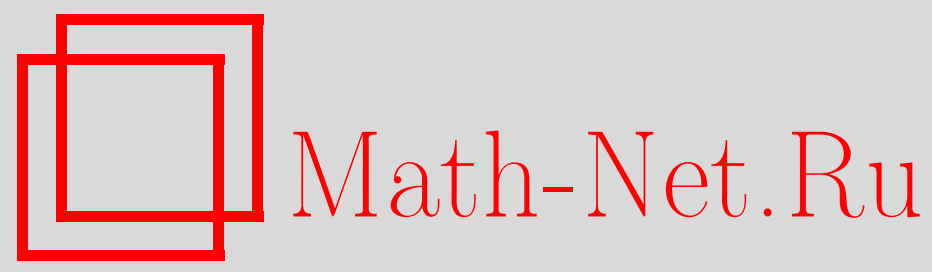

А. В. Пухликов, Бирациональные автоморфизмы алгебраических многообразий с пучком двойных квадрик, Матем. заметки, 2000, том 67, выпуск 2, 241-249

DOI: https://doi.org/10.4213/mzm832

Использование Общероссийского математического портала Math-Net.Ru подразумевает, что вы прочитали и согласны с пользовательским соглашением http://www . mathnet.ru/rus/agreement

Параметры загрузки:

IP: 3.80 .253 .173

26 апреля 2023 г., 13:50:25

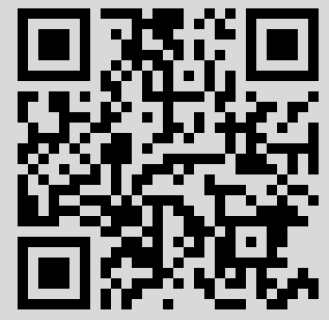




\section{БИРАЦИОНАЛЬНЫЕ АВТОМОРФИЗМЫ АЛГЕБРАИЧЕСКИХ МНОГООБРАЗИЙ С ПУЧКОМ ДВОЙНЫХ КВАДРИК}

\section{А.В. Пухликов}

Доказана бирациональная жесткость многомерных алгебраических многообразий с пучком двойных квадрик индекса 1 , достаточно закрученньм по базе.

Библиографиял: 7 названий.

\section{Введение}

0.1. Рассмотрим Фано-расслоение $\pi: V \rightarrow S$, где $V$ - гладкое проективное многообразие размерности $\geqslant 4$, удовлетворяющее условию $\mathrm{Pic} V=\mathbf{Z} K_{V} \oplus \pi^{*} \operatorname{Pic} S, S$ - гладкое проективное многообразие размерности $\geqslant 1$, и антиканонический дивизор $\left(-K_{V}\right)$ обилен на слоях $\pi$. Общий слой $F_{\eta}$ (т.е. многообразие $V$, рассматриваемое над незамкнутым полем $\mathbf{C}(S))$ есть многообразие Фано. Подгруппу $\operatorname{Bir} F_{\eta} \subset \operatorname{Bir} V$ бирациональных автоморфизмов, сохраняющих слои $\pi$, назовем группой собственны автоморфизмов $Ф$ ано-расслоения $V / S$.

Напомним [1]- [4], что $V / S$ называется бирационально жестким, если для любого бирационального отображения

$$
\chi: V \rightarrow V^{\prime}
$$

на унилинейчатое многообразие $V^{\prime}$ той же размерности выполнено условие монотонности

$$
c\left(D^{*}, V\right) \leqslant c\left(D^{\prime}, V^{\prime}\right) .
$$

Здесь $D^{\prime} \in \Sigma^{\prime}, \Sigma^{\prime}$ - линейная система дивизоров на $V^{\prime}$ без неподвижных компонент, $D^{*} \in \Sigma^{*}, \Sigma^{*}-$ собственньй прообраз $\Sigma^{\prime}$ на $V$ относительно композиции

$$
\chi^{*} \circ \chi: V \rightarrow V^{\prime}
$$

где $\chi^{*} \in \operatorname{Bir} F_{\eta}$ - некоторый (зависящий от $\Sigma^{\prime}$ ) собственньй бирациональньй автоморфизм $V / S$, а

$$
c(Y, X)=\sup \left\{\frac{b}{a}|b \geqslant 1, a \geqslant 1,| a Y+b K_{X} \mid \neq \varnothing\right\}
$$

есть порог канонического присоединения. Если для любых $V^{\prime}, \chi, \Sigma^{\prime}$ можно взять $\chi^{*}=\mathrm{id}$, т.е.

$$
c(D, V) \leqslant c\left(D^{\prime}, V^{\prime}\right)
$$

то Фано-расслоение $V / S$ назьвается бирационально сверхжестким.

Работа выполнена при финансовой поддержке Российского фонда фундаментальных исследований, гранта № 99-01-01132, и Фонда поддержки исследований молодых ученых, грант № 99-15-96013. 
0.2. Цель настоящей работы - доказать бирациональную сверхжесткость многомерных алгебраических многообразий с пучком двойных квадрик. Пусть $S=\mathbf{P}^{1}, \pi: V \rightarrow \mathbf{P}^{1}$ - гладкое многообразие размерности $M+1 \geqslant 5$, каждый слой которого есть двойная квадрика индекса 1. Точнее, имеет место коммутативная диаграмма

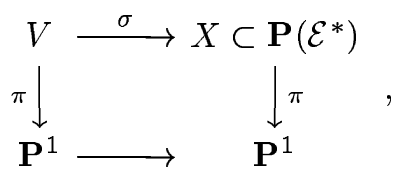

где $\sigma$ - двойное накрытие, $\mathbf{P}\left(\mathcal{E}^{*}\right)$ есть локально-тривиальное $\mathbf{P}=\mathbf{P}^{M+1}$-расслоение над $\mathbf{P}^{1}$, слой $X_{t}=\pi^{-1}(t) \subset \mathbf{P}$ есть $M$-мерная квадрика и дивизор ветвления $\sigma$ высекается на $X_{t}$ гиперповерхностью степени $2(M-1)$ в $\mathbf{P}$ (подробнее конструкцию пучка двойных квадрик см. в п. 1). Основным результатом настоящей работы является

ТЕорема 1. Пусть $V$ - общее многообразие, удовлетворяющее $K^{2}$-условию: численный класс $N K_{V}^{2}-f$ не является әффективным ни для каких $N \in \mathbf{Z}, f-$ класс әффективного дивизора в слое пучка $\pi$. Тогда Фано-расслоение $\pi: V \rightarrow \mathbf{P}^{1}$ является бирачионально сверхжестким.

Для Фано-расслоения $\pi: V \rightarrow \mathbf{P}^{1}$, удовлетворяющего $K^{2}$-условию, сверхжесткость влечет ряд стандартных свойств:

i) единственная нетривиальная структура Фано-расслоения на $V$ - это пучок слоев морфизма $\pi$, иными словами, любое бирациональное отображение

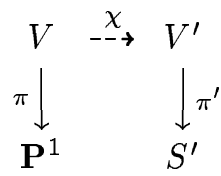

на произвольное $Ф$ ано-расслоение той же размерности является послойным, т.е. существует $\alpha: \mathbf{P}^{1} \rightarrow S^{\prime}$ такое, что $\pi^{\prime} \circ \chi=\alpha \circ \pi$;

ii) любой бирациональньй автоморфизм многообразия $V$ является послойным; для общего $V$ имеет место совпадение $\operatorname{Bir} V=\operatorname{Aut} V=\mathbf{Z} / 2 \mathbf{Z}$;

iii) многообразие $V$ не рационально.

Для тех Фано-расслоений $V \rightarrow \mathbf{P}^{1}$, для которых $X=Q \times \mathbf{P}^{1}, Q \subset \mathbf{P}$ - гладкая квадратичная гиперповерхность, наша теорема была доказана в [2]. Все рассуждения работы [2] годятся и для общего случая произвольного $X$, однако они не исключают наиболее трудного варианта максимальной особенности, лежащей над вершиной конуса $Q_{t} \subset \mathbf{P}$. Именно для того, чтобы избежать особых слоев на $X$, в [2] класс рассматриваемых многообразий был ограничен прямым произведением. Однако теперь усиленная техника гиперкасательных систем позволяет разобрать до конща и случай вырожденных слоев. Доказательство теоремы содержится в п. 2 .

Результаты работ [2], [3], [5] и теорема 1 позволяют предположить, что импликация $\left(K^{2}\right.$-условие $\Rightarrow$ бирациональная жесткость) справедлива для всех многообразий с пучком двойных гиперповерхностей Фано.

ГИПоТЕЗА. Пусть $\pi: V \rightarrow \mathbf{P}^{1}$ есть Фано-расслоение с неособым $V$, слой которого $\pi^{-1}(t)$ двулистно накрывает $Q_{m} \subset \mathbf{P}$ с ветвлением в $W_{2 l}^{*} \cap Q_{m}, l+m=M+1$. 
Предположим, что $V$ удовлетворяет $K^{2}$-условию. Тогда $\pi: V \rightarrow \mathbf{P}^{1}$ бирационально сверхжесткое.

Техника гиперкасательных систем позволяет продвинуть доказательство гипотезы (по традиционной схеме метода максимальньх особенностей) достаточно далеко. Точнее, исключаются все возможности для максимальной особенности, кроме одной - когда ее центр на $V$ есть особая точка слоя $\pi$, происходящая из особенности на дивизоре ветвления (если особенность происходит из двойной точки гиперповерхности $Q_{t} \subset \mathbf{P}^{M}$, то успешно работают рассуждения [2]). Для завершения доказательства необходимо дальнейшее совершенствование техники.

0.3. Настоящая работа является непосредственным продолжением [2], [3]. Двойные пространства и двойные квадрики индекса 1 имеют малую степень и потому наиболее удобны для экспериментов по изучению бирациональной геометрии методом максимальных особенностей. Именно эти многообразия дали первые примеры бирационально (сверх)жестких многообразий Фано произвольной размерности [5].

Новая техника изучения Фано-расслоений, развитая в [3], позволила сразу доказать сверхжесткость многообразий с пучком двойных пространств ([3, п. 8]). Однако степень 4 оказалась слишком велика для того, чтобы автоматически получить аналогичный результат и для двойных квадрик, хотя было очевидно, что такой результат справедлив. Методов работы [3] хватило лишь на двойные накрытия прямых произведений [2], однако исключить максимальную особенность над вершиной конуса не удавалось.

Работа [2] была фактически выполнена в 1997 г. во время моего пребьвания в Математическом институте им. Макса Планка в Бонне, ФРГ. Там же была развита и техника гиперкасательных систем, модификация которой позволила доказать основную теорему настоящей статьи. Пользуясь случаем, хочу поблагодарить Институт за прекрасные условия работы.

\section{1. Многообразия с пучком двойных квадрик}

1.1. Конструкция. Мы изучаем гладкие проективные $(M+1)$-мерные многообразия $V$ над полем комплексных чисел $\mathbf{C}, M \geqslant 4$, допускающие морфизм $\pi: V \rightarrow \mathbf{P}^{1}$, слои которого - двойные квадрики индекса 1. Точнее, пусть

$$
\mathcal{E}=\bigoplus_{i=0}^{M+1} \mathcal{O}_{\mathbf{P}^{1}}\left(a_{i}\right)
$$

- локально-свободный пучок ранга $M+2$ на $\mathbf{P}^{1}$, где для удобства предполагаем, что $0=a_{0} \leqslant a_{1} \leqslant \cdots \leqslant a_{M+1}$. Его проективизация $X=\operatorname{Proj}(\mathcal{E})=\mathbf{P}\left(\mathcal{E}^{*}\right)$ есть $\mathbf{P}^{M+1}$-расслоение над прямой. Проекцию на базу обозначим тем же символом $\pi$.

Пусть $G$ - слой морфизма $\pi$; тогда $\mathrm{Pic} X=\mathbf{Z} L \oplus \mathbf{Z} G$, где $L$ - класс тавтологического пучка $\mathcal{O}_{X / \mathbf{P}^{1}}(1)$, т.е. $\pi_{*} \mathcal{O}_{X}(L)=\mathcal{E}$. В силу сделанного вьше предположения пучок $\mathcal{O}_{X}(L)$ порожден своими сечениями, т.е. линейная система $|L|$ свободна. Канонический класс многообразия $X$ есть

$$
K_{X}=-(M+2) L+\left(\sum_{i=1}^{M+1} a_{i}-2\right) G
$$


Рассмотрим на $X$ гладкий дивизор $Q \in|2 L+\mu G|, \mu \in \mathbf{Z}$. Его слои $Q_{t}=Q \cap \pi^{-1}(t)$ над точками $t \in \mathbf{P}^{1}$ суть квадратичные гиперповерхности. Канонический класс многообразия $Q$ есть

$$
K_{Q}=\left.\left(-M L+\left(\sum_{i=1}^{M+1} a_{i}+\mu-2\right) G\right)\right|_{Q} .
$$

Рассмотрим теперь на $X$ дивизор Вейля $W \in|2(M-1) L+2 \lambda G|$ такой, что $W \cap Q-$ гладкий дивизор на $Q$.

Пусть $V$ - (гладкое) двойное накрытие $\sigma: V \rightarrow Q$, разветвленное над $W \cap Q$. Построенное семейство гладких многообразий $V$ обозначим символом $\mathcal{V}\left(a_{*}, \mu, \lambda\right)$. Проекцию $V$ на $\mathbf{P}^{1}$ обозначаем символом $\pi$, а ее слой - символом $F_{t}, t \in \mathbf{P}^{1}$. Очевидно, $\operatorname{Pic} V=\mathbf{Z} K_{V} \oplus \mathbf{Z} F$ и морфизм $\pi: V \rightarrow \mathbf{P}^{1}$ расслаивает $V$ на многообразия Фано индекca 1 , степени 4 . Канонический класс многообразия $V$ имеет вид

$$
K_{V}=-\sigma^{*}\left(\left.L\right|_{Q}\right)+\left(\sum_{i=1}^{M+1} a_{i}+\mu+\lambda-2\right) F .
$$

1.2. Условие общности положения. Мы будем предполагать, что выполнены следующие три условия общности положения.

(G1) Если $Q_{t} \subset \mathbf{P}^{M+1}$ - особая квадрика, то $Q_{t}$ имеет ровно одну особую точку, т.е. $Q_{t}$ - конус над неособой квадрикой в $\mathbf{P}^{M}$.

(G2) Если многообразие $W \cap Q_{t}$ имеет особенности (это может случиться лишь для конечного числа точек $t \in \mathbf{P}^{1}$ ), то $Q_{t}$ - неособая квадрика, множество $\operatorname{Sing} W \cap Q_{t}$ конечно и каждая особенность $x \in W \cap Q_{t}$ есть невырожденная квадратичная особенность. Более того, квадратичные касательные конуса

$$
T_{x}\left(T_{x} Q_{t} \cap Q_{t}\right) \text { и } T_{x}\left(W \cap Q_{t}\right)
$$

находятся в общем положении, т.е. пересечение их проективизаций в проективном пространстве $\mathbf{P}\left(T_{x} Q_{t}\right) \cong \mathbf{P}^{M-1}$ есть неособое многообразие размерности $M-3$ и степени 4 .

(G3) Если $Q_{t} \subset \mathbf{P}^{M+1}$ - квадратичньй конус с вершиной в точке $x$, то рассмотрим линейную систему координат $\left(z_{1}, \ldots, z_{M+1}\right)$ на $\mathbf{C}^{M+1} \subset \mathbf{P}^{M+1}$ с центром в точке $x$, и пусть $q=0$ и $g=w_{0}+w_{1}+\cdots+w_{2(M-1)}$ - уравнения квадрики $Q_{t}$ и гиперповерхности $W_{t}=W \cap G_{t}$ соответственно. Прежде всего, $w_{0} \neq 0$, т.е. $x \notin W$. В дальнейшем будем предполагать, что $w_{0}=1$.

Рассмотрим степенной ряд $(1+a)^{1 / 2}=1+\sum \gamma_{i} a^{i}=1+\frac{1}{2} a+\cdots$, где $a-$ формальная переменная. Положим

$$
\begin{aligned}
\sqrt{g}=\left(1+w_{1}+\cdots+w_{2(M-1)}\right)^{1 / 2} & =1+\sum_{i=1}^{\infty} \gamma_{i}\left(w_{1}+\cdots+w_{2(M-1)}\right)^{i} \\
& =1+\sum_{i=1}^{\infty} \Phi_{i}\left(w_{1}, \ldots, w_{2(M-1)}\right)
\end{aligned}
$$

где $\Phi_{i}\left(w_{1}\left(z_{*}\right), \ldots, w_{2(M-1)}\left(z_{*}\right)\right)$ однородны по $z_{*}$ степени $i$. Очевидно, $\Phi_{i}\left(w_{*}\right)=w_{i}+\cdots$ при $i \leqslant 2(M-1)$, где многоточие обозначает многочлен от $w_{1}, \ldots, w_{i-1}$. Положим для $k \geqslant 1$

$$
[\sqrt{g}]_{k}=1+\sum_{i=1}^{k} \Phi_{i}\left(w_{*}\right), \quad g^{(k)}=g-[\sqrt{g}]_{k}^{2} .
$$


Легко видеть, что $g^{(k)}$ начинается с однородной компоненты степени $k+1$ по $z_{*}$. Эта компонента при $k+1 \leqslant 2(M-1)$ имеет вид $w_{k+1}+\cdots$, где многоточие обозначает многочлен от $w_{1}, \ldots, w_{k}$.

Наше условие общности положения (G3) теперь формулируется так: для некоторой системы координат $z_{*}$ набор многочленов

$$
q, g^{(M-1)}, \ldots, g^{(2 M-3)}
$$

образует регулярную последовательность в $\mathcal{O}_{x, \mathbf{P}}{ }^{M+1}=\mathcal{O}_{x, \mathbf{C}^{M+1}}$, и, более того, в окрестности точки $x$ система уравнений

$$
q=g^{(M-1)}=\cdots=g^{(2 M-4)}=0
$$

задает поверхность, ни одна компонента которой не содержится в гиперплоскости, содержащей точку $x$. Иньми словами, если $l(z)$ - произвольная линейная форма, то последовательность

$$
l, q, g^{(M-1)}, \ldots, g^{(2 M-4)}
$$

остается регулярной в $\mathcal{O}_{x, \mathbf{P}} \mathbf{P}$. При $M=4$ мы дополнительно требуем, чтобы для любой линейной формы $l(z)$ схема $l=q=g^{(3)}=g=0$ была приведена в общей точке.

\section{2. Доказательство теоремы $1^{*}$}

2.1. Метод максимальных особенностей. Основные понятия, конструкции и теоремы метода многократно публиковались, начиная с исходной работы В. А. Исковских и Ю. И. Манина о квартике [6], см. [1], [3], [7]. Поэтому нет необходимости воспроизводить здесь детали. Для $\Phi$ ано-расслоений над $\mathbf{P}^{1}$ техника метода максимальных особенностей была развита в [3] и затем использована в [2], [4]. В данной работе мы сформулируем достаточное условие сверхжесткости в терминах степеней и кратностей алгебраических циклов и проверим его для наших Фано-расслоений. Детали доказательства достаточного условия читатель найдет в [2]-[4].

Пусть $\pi: Y \rightarrow \mathbf{P}^{1}$ - гладкое Фано-расслоение с $\mathrm{Pic} Y=\mathbf{Z} K_{Y} \oplus \pi^{*} \mathrm{Pic} \mathbf{P}^{1}$, удовлетворяющее $K^{2}$-условию, т.е. численньй класс $N K_{Y}^{2}-f$, где $f \in A^{2} Y$ - класс гиперплоского сечения слоя $F_{t}=\pi^{-1}(t)$, не представлен эффективным циклом коразмерности 2 ни для какого $N \in \mathbf{Z}$. Следуя [2], [3], мы называем неприводимьй цикл $B \subset Y$ горuзонтальным. если он накрывает базу, $\pi(B)=\mathbf{P}^{1}$, и вертикальным, если он лежит в слое $\pi$. $C$ тепенъю вертикального цикла $B$ назовем число

$$
\operatorname{deg} B=\left(B \cdot\left(-K_{Y}\right)^{\operatorname{dim} B}\right)>0 \text {. }
$$

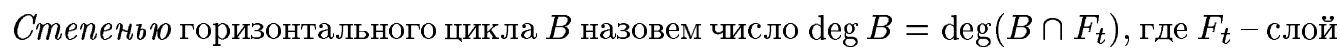
общего положения.

ПРЕДЛОЖЕНИЕ 1 (достаточное условие бирациональной сверхжесткости). Предположим, что для любой подвижной линейной системы $\Sigma \subset\left|-n K_{Y}+l F\right|$, любого неприводимого вертикального иикла $A \subset F_{t}$ коразмерности 2 (относительно $Y$ ), любого неприводимого горизонтального иикла $B \subset Y$ коразмерности 2, любого вертикального чикла $C \subset F_{t}$ коразмерности 3 (относительно $Y$ ) и любой точки $x \in F_{t}$ выполнены следуюшие условия:

i) $\operatorname{mult}_{A} \Sigma \leqslant n, \operatorname{mult}_{B} \Sigma \leqslant n$;

ii) $\operatorname{mult}_{x} C \leqslant(4 / \operatorname{deg} Y) \operatorname{mult}_{x} F_{t} \operatorname{deg} C$; 
iii) $\operatorname{mult}_{x} A \leqslant\left(2\right.$ mult $\left._{x} F_{t} / \operatorname{deg} Y\right) \operatorname{deg} A, \operatorname{mult}_{y} \widetilde{A} \leqslant(2 / \operatorname{deg} Y) \operatorname{deg} A$, zде $y \in E_{x} \subset \widetilde{F}_{t}$ - произвольная бесконечно близкая точка над $x \in F_{t}, \widetilde{A}-$ собственный прообраз А при раздутии точки $x, \varphi: \widetilde{F}_{t} \rightarrow F_{t}, E_{x}=\varphi^{-1}(x)$.

Тогда Фано-расслоение $\pi: Y \rightarrow \mathbf{P}^{1}$ бирационально сверхжесткое.

Здесь $\operatorname{deg} Y=\left(F \cdot\left(-K_{Y}\right)^{\operatorname{dim} Y-1}\right)$ в соответствие с данньм вьше определением степени.

ДоказАтельство см. в [2]-[4]. Схема рассуждений такова: если $Y \rightarrow \mathbf{P}^{1}$ не является бирационально сверхжестким, то на $Y$ имеется подвижная линейная система $\Sigma \subset\left|-n K_{Y}+l F\right|$, имеющая максимальную особенность $\nu$. Если центр максимальной особенности имеет коразмерность 2 , то получаем противоречие с условием і). Пусть $x \in F_{t}$ - точка, принадлежащая центру. Рассмотрим эффективный цикл $Z=\left(D_{1} \bullet D_{2}\right)$, $D_{i} \in \Sigma$, и разобьем его на вертикальную и горизонтальную компоненты

$$
Z=Z^{h}+Z^{v}=Z^{h}+\sum_{s \in \mathbf{P}^{1}} Z_{s}^{v},
$$

где носитель цикла $Z_{s}^{v}$ содержится в слое $F_{s}$. Отметим, что в силу условия iі)

$$
\operatorname{mult}_{x} Z^{h} \leqslant 4 n^{2}
$$

В самом деле, рассмотрим эффективньй цикл $C=\left(Z^{h} \bullet F_{t}\right)$. Очевидно, mult $_{x} C \geqslant$ mult $_{x} Z^{h}$ mult $_{x} F_{t}$, в то время как $\operatorname{deg} Z^{h}=\operatorname{deg} C=n^{2} \operatorname{deg} Y$. Применяя ii), получаем неравенство (1). Далее, в силу условия іiі) имеем

$$
\operatorname{mult}_{x} Z_{t}^{v} \leqslant 4 n l
$$

Считая максимальную особенность $\nu$ сверхмаксимальной [2], [3] и рассуждая, как в [2], $[3]$, получаем противоречие.

Итак, для доказательства теоремы $1^{*}$ достаточно проверить справедливость условий і)-іiі) для Фано-расслоения $\pi: V \rightarrow \mathbf{P}^{1}$.

2.2. Максимальные циклы. Условие і) было фактически проверено в [2]. Поскольку линейная система на гладкой двойной квадрике размерности $\geqslant 4$ не может иметь максимальных особенностей [5], подвижная линейная система $\Sigma$ на $V$ не может иметь максимальных особенностей, накрьвающих базу, т.е. mult $_{B} \Sigma \leqslant n$ для любого горизонтального цикла $B \subset V$ коразмерности 2. Далее, для любого простого дивизора Вейля $A \subset F_{t}$ имеем: $\sigma(A) \subset Q_{t}-$ простой дивизор Вейля на квадрике $Q_{t}$. Дивизоры семейства $\sigma\left(\left.\Sigma\right|_{F_{t}}\right)$ высекаются на $Q_{t}$ гиперповерхностями степени $2 n$, так что если $\operatorname{mult}_{A} \Sigma>n$, то $\sigma(A)=H \cap Q_{t}$ - гиперплоское сечение, причем морфизм $\sigma: A \rightarrow \sigma(A)$ бирационален. Значит, теоретико-схемное пересечение $H \cap Q_{t} \cap W$ не приведено в каждой точке. Однако такого не может быть: по лемме 3.4.2 из [7] dim $\operatorname{Sing} H \cap Q_{t} \cap W \leqslant 1$. Противоречие.

Этим условие і) доказано.

2.3. Проверка условия ii). Очевидно, $\operatorname{deg} V=4$, так что іi) принимает вид неравенства

$$
\operatorname{mult}_{x} C \leqslant \operatorname{mult}_{x} F_{t} \operatorname{deg} C,
$$

которое, конечно, выполнено, так как верно более сильноенеравенство mult $x$ $C \leqslant \operatorname{deg} C$. 
2.4. Проверка условия iii). Если $z=\sigma(x)$ - гладкая точка квадрики $Q_{t}$, то дословное повторение рассуждений работы [2] доказывает іiі). Схема доказательства такова. Имеются две возможности: $\sigma(A)=T_{z} Q_{t} \cap Q_{t}$ и $\sigma(A) \neq T_{z} Q_{t} \cap Q_{t}$. В первом случае iii) проверяется непосредственно с использованием (G1), (G2) и упомянутой выше леммы 3.4.2 из [7]. Во втором случае іiі) получается прменением уже доказанного неравенства іi) к эффективному циклу $\left(A \bullet \sigma^{-1}\left(T_{z} Q_{y} \cap Q_{t}\right)\right)$ коразмерности 2.

Пусть $z=\sigma(x)$ - вершина конуса $Q_{t}$. Прежде всего, отметим, что первое из двух неравенств iii) в этом случае тривиально: оно утверждает, что mult $x A \leqslant \operatorname{deg} A$. Предположим, что для простого дивизора Вейля $A \subset F_{t}$ не вьполнено второе неравенство:

$$
\operatorname{mult}_{y} \widetilde{A}>\frac{1}{2} \operatorname{deg} A,
$$

где $y \in E_{x}=\varphi^{-1}(x) \subset \widetilde{F}_{t}-$ бесконечно близкая точка первого порядка над $x, \varphi: \widetilde{F}_{t} \rightarrow F_{t}$ - раздутие точки $x$. Покажем, что сделанное предположение приводит к противоречию. Сделаем это в три шага.

ШАГ 1. Точка $z=\sigma(x)$ не лежит на дивизоре ветвления, так что $\sigma(y)$ - корректно определенная бесконечно близкая точка первого порядка над $z$, определяющая прямую $(z, \sigma(y)) \subset Q_{t}$. Через $T_{y} Q_{t}$ обозначим гиперплоскость в $\mathbf{P}$, которая касается $Q_{t}$ вдоль этой прямой. Положим $T=\sigma^{-1}\left(T_{y} Q_{t} \cap Q_{t}\right) \subset F_{t}$. Очевидно, $\operatorname{mult}_{y} \widetilde{T}=2, \operatorname{deg} T=4$, так что условие iii) для $T$ вьполнено. В частности, $A \neq T$.

ШАГ 2. Положим $B=E_{x} \cap \widetilde{T}$ (если рассматривать $T_{y} Q_{t} \cap Q_{t}$ как аффинный конус с вершиной $x$, то $B$ - его проективизация).

ЛЕмма 1. Имеет место неравенство

$$
\operatorname{mult}_{B} \widetilde{A} \geqslant \frac{1}{4}\left(2 \operatorname{mult}_{y} \widetilde{A}-\operatorname{mult}_{x} A\right) .
$$

ДоКАЗАТЕЛЬСТВО: см. [4], п. 3.6.

Рассмотрим теперь алгебраический цикл $(A \bullet T)=C$. Нетрудно проверить, что $C$ удовлетворяет неравенству

$$
\operatorname{mult}_{x} C>\frac{3}{4} \operatorname{deg} C .
$$

ШАГ 3. Покажем, что этого не может быть. Предположим сначала, что $M \geqslant 5$. Очевидно, цикл $C$ можно считать неприводимьм. Пусть $H=T_{y} Q_{t} \cong \mathbf{P}^{M}$ - касательная гиперплоскость, $\sigma: R \rightarrow H$ - двойное накрытие, разветвленное над гиперповерхностью $W_{H}=W \cap H$. Многообразие $R$ неприводимо и, вообще говоря, имеет особенности. Цикл $T$ естественно отождествляется с дивизором в $R$. Поэтому и $C$ можно отождествить с циклом коразмерности 2 в $R$. В силу этого отождествления мы пишем $x \in R$ и т.д.

Пусть $P \in \operatorname{Pic~} R$ - класс гиперплоского сечения, т.е. $\mathcal{O}_{R}(P) \cong \sigma^{*} \mathcal{O}_{H}(1), H \hookrightarrow \mathbf{P}$. Определим $k$-ую гиперкасательную систему в точке $x \in R$ условием

$$
\Lambda_{k}=\left\{D \in|k P| \mid \operatorname{mult}_{x} D \geqslant k+1\right\} .
$$

ЛЕмма 2. Гиперкасательные линейные системы $\Lambda_{k}$ непусты при $k=M-1$, .., $2 M-3$. Более того,

$$
\operatorname{codim}_{T} \operatorname{Bs}\left(\left.\Lambda_{M-2+i}\right|_{T}\right) \geqslant i
$$

nрu $i=1, \ldots, M-2$. 
ДоКАЗАТЕЛЬСтво. Это немедленно вытекает из условия (G3), где в качестве гиперплоскости, содержащей точку $p=\sigma(x) \in Q_{t} \cap H$, берется $H$. В самом деле, пусть $z_{1}, \ldots, z_{M+1}-$ система линейных координат с центром в $p$, причем $\mathbf{P} \supset H=\left\{z_{M+1}=0\right\}$. Пусть $U=\mathbf{C}_{\left(z_{1}, \ldots, z_{M}\right)}^{M}$. Над афффинным множеством $U$ многообразие $R$ задается уравнением $y^{2}=g\left(z_{1}, \ldots, z_{M}, 0\right)$ в $\mathbf{C}_{(y)} \times U$. Координата $y$ определяет сечение пучка $\mathcal{O}_{R}((M-1) P)$, поэтому регулярные на $\mathbf{C} \times U$ функции

$$
f_{k}=y-[\sqrt{g}]_{k}
$$

определяют при $k \geqslant M-1$ сечения пучков $\mathcal{O}_{R}(k P)$ соответственно. Более того, при $k \geqslant M-1$ положим

$$
\Delta_{k}=\overline{\left\{f_{i}=0\right\}} \subset R .
$$

Легко видеть, что $\Delta_{k} \in \Lambda_{k}$. Образ дивизора $\Delta_{k}$ на $U=\mathbf{C}^{M}$ задается уравнением $g^{(k)}\left(z_{1}, \ldots, z_{M}, 0\right)=0$. Теперь применяем (G3) и получаем утверждение нашей леммы.

Пусть $D_{i} \in \Lambda_{M-2+i}, i=1, \ldots, M-1,-$ обшие дивизоры. Построим цепочку неприводимых циклов $C_{2}, C_{3}, \ldots, C_{M-1} \subset R, \operatorname{codim} C_{j}=j$, следующим образом. Положим $C_{2}=C$. Предположим, что $C_{j}, j=2, \ldots, l$, уже построены и $l \leqslant M-2$. В силу леммы 2 общий дивизор $D_{l}$ не содержит $C_{l}$. В силу определения гиперкасательной системы существует неприводимая компонента $Z$ цикла $\left(C_{l} \bullet D_{l}\right)$, удовлетворяющая неравенству

$$
\frac{\operatorname{mult}_{x}}{\operatorname{deg}} Z \geqslant \frac{\operatorname{mult}_{x}}{\operatorname{deg}} C_{l} \cdot \frac{M-1+l}{M-2+l} .
$$

Положим $C_{l+1}=Z$.

Цикл $C_{M-1} \subset R$ есть неприводимая кривая. Имеем оценку

$$
\begin{gathered}
\frac{\text { mult }_{x}}{\operatorname{deg}} C_{M-1}>\frac{3}{4} \cdot \frac{M+1}{M} \cdot \frac{M+2}{M+1} \cdots \cdots \frac{2 M-3}{2 M-4} \\
\frac{3(2 M-3)}{4 M}
\end{gathered}
$$

и легко видеть, что $3(2 M-3) /(4 M) \geqslant 1$, что дает противоречие.

Предположим, что $M=4$. Здесь линейная система $\Lambda_{3}$ состоит из единственного дивизора $D_{3}$. Имеются две возможности: либо $C \subset D_{3}$, либо $C \not \subset D_{3}$. В первом случае условие іiі) вытекает из условия общности положения, ибо $C=T \cap D_{3}$. В противном случае цикл $C_{3}=\left(C \bullet D_{3}\right)$ эффективен. Однако он удовлетворяет неравенству (mult $x / \operatorname{deg}) C_{3}>1$, что невозможно.

Этим свойство iii) и теорема $1^{*}$ полностью доказаны.

\section{3. Трехмерный случай}

Бо́льшая часть проведенных рассуждений работает без каких-либо изменений и при $M=3$. Исключение составляют два "подводных камня". Во-первых, размерность уже не позволяет пользоваться леммой 3.4.2 из [7]. Однако дополнительные условия общности положения многообразия $V$ заменяют эту лемму. Читатель без труда восстановит необходимые детали.

Вторая сложность более существенна: на $V$ в размерности три могут появиться горизонтальные максимальные циклы! Это связано, конечно, с тем, что на гладкой двойной квадрике индекса 1 размерности 3 любая прямая вне дивизора ветвления может быть 
максимальной особенностью [7] и, соответственно, группа бирациональных автоморфизмов этого многообразия весьма велика (она была описана В. А. Исковских в середине 70-х гг.).

Пусть $\mathcal{L}(V)$ - (квазипроективное) многообразиепрямьх в слоях морфизма $\pi: V \rightarrow \mathbf{P}^{1}$, не лежащих на дивизоре ветвления,

$$
\mathcal{L}(V)=\left\{L \subset F_{t} \mid\left(L \cdot K_{V}\right)=-1, \sigma(L) \not \subset W\right\} .
$$

Естественную проекцию $\mathcal{L}(V) \rightarrow \mathbf{P}^{1}$ обозначим тем же символом $\pi$. Каждое сечение (определенное, вообщеговоря, вне конечного множества точек) $s: \mathbf{P}^{1} \rightarrow \mathcal{L}(V), \pi \circ s=\mathrm{id}$, определяет послойньй бирациональньй автоморфизм $\tau_{s} \in \operatorname{Bir} F_{\eta} \subset \operatorname{Bir} V$, где $F_{\eta}-$ слой над общей точкой, многообразие Фано над незамкнутьм полем $\mathbf{C}\left(\mathbf{P}^{1}\right)$. Инволюции $\tau_{s}$ свободно порождают в $\operatorname{Bir} V$ нормальную подгрупшу

$$
B(V)=\underset{\substack{s: \mathbf{P}^{1} \rightarrow \mathcal{L}(V) \\ \pi \circ s=\mathrm{id}}}{*}\left\langle\tau_{s}\right\rangle,
$$

см. [7], причем имеет место точная последовательность

$$
1 \rightarrow B(V) \rightarrow \operatorname{Bir} V \rightarrow \text { Aut } V \rightarrow 1
$$

где группа Aut $V$ конечна, а в общем случае изоморфна $\mathbf{Z} / 2 \mathbf{Z}$.

Дословное повторение рассуждений п. 2, с учетом сделанных замечаний, приводит к следующему результату.

Теорема 2. Общее четырехмерное Фано-расслоение $V$, удовлетворяющее $K^{2}-y с-$ ловию, есть бирачионально жсесткое многообразие, группа бирачиональных автоморфизмов которого описывается точной последовательностью (3).

Возникает вопрос: существуют ли сечения $s: \mathbf{P}^{1} \rightarrow \mathcal{L}(V)$ для общего Фано-расслоения $V$ ? Ответ автору неизвестен. По-видимому, он отрищателен. Если он действительно отрицателен, то и для $M=3$ мы получаем бирациональную сверхжесткость.

\section{СПИСОК ЦИТИРОВАННОЙ ЛИТЕРАТУРЫ}

[1] Pukhlikov A. V. Essentials of the method of maximal singularities, Warwick Preprint № $31 / 1996$.

[2] Pukhlikov A. V. Certain examples of birationally rigid varieties with a pencil of double quadrics // J. Math. Sci. 1999. V. 94. № 1. P. 986-995.

[3] Пухликов А.В. Бирациональные автоморфизмы трехмерных алгебраических многообразий с пучком поверхностей Дель Пеццо // Изв. РАН. Сер. матем. 1998. Т. 62. № 1. С. 123-164.

[4] Pukhlikov A. V. Birationally rigid Fano fibrations. MPI-preprint, 1998-113.

[5] Пухликов А. В. Бирациональные автоморфизмы двойного пространства и двойной квадрики // Изв. АН СССР. Сер. матем. 1988. Т. 52. №1. С. 229-239.

[6] Исковских В. А., Манин Ю. И. Трехмерные квартики и контрпримеры к проблеме Люрота // Матем. сб. 1971. Т. 86. №1. С. 140-166.

[7] Iskovskikh V. A., Pukhlikov A. V. Birational automorphisms of multi-dimensional algebraic varieties // J. Math. Sci. 1996. V. 82. P. 3528-3613.

Математический институт им. В. А. Стеклова РАН 